\title{
Development of a Love-wave Immunosensor with Microfluidic Technology to Detect Phages in Dynamic Mode \\ D. Matataqui ${ }^{1}$, D. Moynet ${ }^{2}$, M.J. Fernández ${ }^{1}$, J. Fontecha ${ }^{1}$, J.P. Esquivel ${ }^{3}$, I. Gràcia ${ }^{3}$, C. Cané ${ }^{3}$, C. Déjous $^{4}$, D. Rebière ${ }^{4}$, J.P. Santos ${ }^{1}$, M.C. Horrillo ${ }^{1}$ \\ ${ }^{1}$ GRIDSEN, CSIC, Serrano 144, 28006 Madrid, Spain \\ Corresponding e-mail address: d.m@csic.es \\ ${ }^{2}$ Université de Bordeaux, Laboratoire d'immunologie, Bordeaux 33706, France \\ ${ }^{3}$ Instituto de Microelectrónica de Barcelona, CSIC, Campus UAB, 08193 Bellaterra, Spain \\ ${ }^{4}$ Université de Bordeaux, IMS, IPB-ENSEIRB, CNRS UMR 5218, Talence 33405, France
}

\begin{abstract}
:
The goal has been to develop an immunosensor based on a Love-wave device and a PDMS microfluidic chip in order to detect potentially pathogenic microorganisms in real time. Therefore, it has been tested with the phage M13 detected through the mouse monoclonal antibody anti-M13 (AM13). The Double-layer Agar (DLA) technique has been used to quantify the number of phages, linked to the sensor surface, in plaques-forming unit (pfu); that has allowed us to compare the number of phages determined by DLA and the estimated by the frequency shift, and both were very similar. With the aim to obtain a short response time, the detection of the phage was carried out in dynamic mode. The range tested concentrations has varied between $2 \times 10^{10} \mathrm{pfu} \cdot \mathrm{ml}^{-1}$ and $5 \times 10^{8} \mathrm{pfu} \cdot \mathrm{ml}^{-1}$. A fast response and a good discrimination among concentrations have been achieved. Besides, the AM13 has been used as secondary antibody in order to measure lower concentrations, such as $5 \cdot 10^{7} \mathrm{pfu} \cdot \mathrm{ml}^{-1}$.
\end{abstract}

Key words: Acoustic wave sensor, Love wave, microfluidic, M13 virus, immunodetection, biosensor.

\section{Introduction}

In order to minimize the transfer of phages among hosts and avoid spread of diseases, the phages have to be detected in a short time, at low concentrations and with an efficient identification. Today, the standard laboratory diagnostic tests for phages are based on their culture, which is very sensitive but cumbersome and time-consuming. On the other hand polymerase chain reaction (PCR) technique is very widespread and it can be run in 30 minutes but is difficult to do out of very clean labs and you need very well trained people to run it. This leads to an urgent need for real-time and in situ detectors.

The systems based on immunoreaction of antibody-antigen binding recognition (also called "immunosensors") generally rely on highly sensitive devices to translate the biological recognition event into a physical magnitude variation in real time. On the other hand, the acoustic wave (AW) sensors are reusable [1], with high sensitivity [2] and cheap systems of measure. Therefore an immunosensor based on AW devices with very high sensitivity can be a good solution in the detection of virus in real time.

Microfluidics technology allows use small volumes of phage samples, and operating in dynamic mode the reaction between antigen and antibody is promoted.

\section{Materials and Methods}

M13 is a filamentous bacteriophage (a phage that infects a bacteria) non-pathogenic for humans [1,2] which is $900 \mathrm{~nm}$ long, $9 \mathrm{~nm}$ of diameter and a weight of $3.1 \times 10^{-18} \mathrm{Kg}$. It is composed of DNA encapsulated in a coating that consists of 2800 copies of the protein $\mathrm{P} 8$ and five of P3, P6, P7 and P9. The protein P3 attaches to the receptor, at the tip of the $F$ pilus of the host Escherichia coli, infecting the bacteria and causing plaques.

The AM13 is a monoclonal antibody from mouse that is used in the immunoreaction to link the phage because it has great affinity with the protein P8. In order to validate the experiment, other monoclonal antibody with lower affinity, goat anti-mouse (GAM), was used as control.

Our Love-wave sensors are based on a shear horizontal surface acoustic wave propagated on ST-cut quartz and guided in a $3.5 \mu \mathrm{m}$ film of $\mathrm{SiO}_{2}$, being the synchronous frequency 163 $\mathrm{MHz}$.

For the manufacture of the PDMS chip, the negative photoresist SU-8 was spun on a clean wafer with a $150 \mu \mathrm{m}$ thickness, and then it is 
exposed to an optical lithography process and baked to obtain the master. The silicone base and curing agent are mixed in a 10:1 ratio in weight. The mixture is degassed to remove any bubbles and poured over the master, next is degassed again, ensuring all entrapped gases are evacuated. After baking and cooling, the PDMS is easily peeled and cut. The microstructure of PDMS and the Love wave device form microchannels of $150 \mu \mathrm{m}$ of height. Previously, Comsol software was used to simulate the flow of the liquid due to microchannel shape in order to achieve a uniform velocity in the path between the IDTs (Fig. 1).

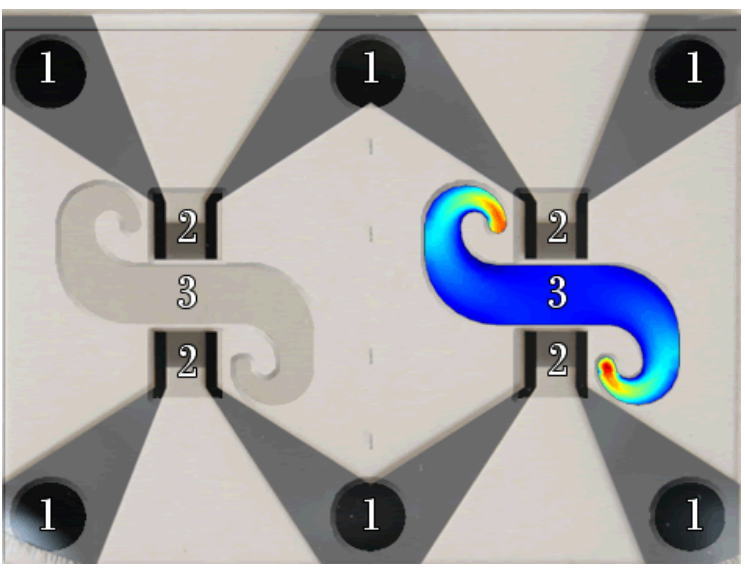

Fig. 1. Love-wave device with two delay lines and microfluidic chip. 1) contacts, 2) IDTs and 3) microchannels (at the right, the simulation of velocity, being the color red the highest velocity and the blue the lowest one).

Our sensors contain two delay lines (DL) and each one works in an oscillator system, which includes an amplifier and a directional coupler. Therefore, when the sensor is perturbed, the oscillating frequency is shifted in order to satisfy the law of Barkhausen, what means that the phase of the closed loop is $2 \pi n$ and the gain is $\mathrm{O}^{+} \mathrm{dB}$. The measured quantity is the output frequency.

The sensors were electrically characterized by means of a vector network analyzer (Agilent 5070B) and the oscillator through a spectrum analyzer (Agilent 9320A).

Love wave sensors are really sensible to the temperature. Therefore in order to keep it constant at $30^{\circ} \mathrm{C}$, a Peltier device controlled by a PID program from the computer was used.

The experiment control and data acquisition in real time were implemented with a PC by means of software made at home. The setup of the experiment is shown in Fig. 2.

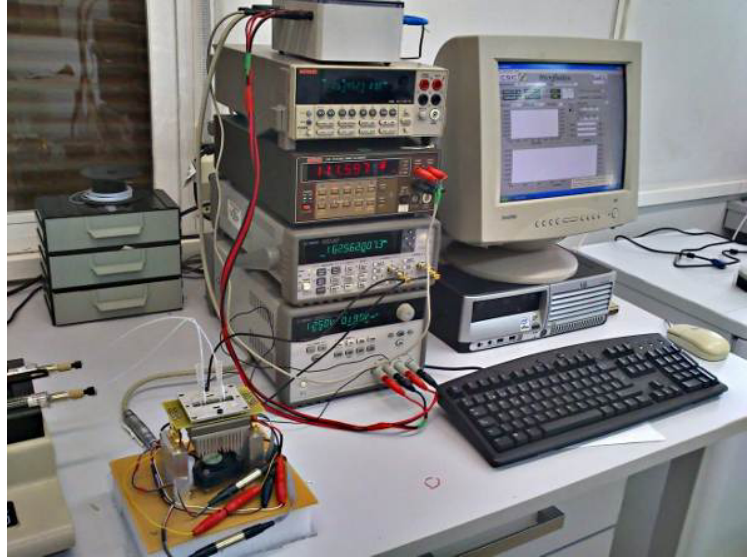

Fig. 2. Experimental setup.

The sensor surface was functionalized according to the following steps:

- First, the oxidation of $\mathrm{SiO}_{2}$ surface was performed depositing fresh piranha etch $\left(\mathrm{H}_{2} \mathrm{SO}_{4}: \mathrm{H}_{2} \mathrm{O}_{2}, 3: 1\right.$, v/v) for $5 \mathrm{~min}$. After rinsing in water, the surface was hydrophilic.

- The Love-wave device was immersed in a freshly prepared $20 \mathrm{mM}$ 3-aminopropyl triethoxysilane (APTES) solution in toluene for 1 hour (15 minutes in sonication, 15 minutes was left in rest, 15 minutes in sonication and 15 minutes was left in rest) followed by a thorough cleaning in toluene and isopropanol. The APTES step covered the surface with amine-terminated silane organic molecules for the subsequent steps.

- Next, the APTES-modified surface reacted with a $20 \mathrm{mM}$ glutaraldehyde (GA) solution for $1 \mathrm{~h}$, followed by rinsing with water and drying with nitrogen. GA was used as a homo-bifunctional cross-linker between the amine groups of the APTES and the primary amines of the immunoglobulins.

The measurement protocol (Fig. 3) was:

- Tris Buffered Saline (TBS) was introduced for 15 minutes to stabilize the frequency, the rate was $10 \mu \mathrm{l} / \mathrm{min}$.

- Then the antibodies $\left(50 \mu \mathrm{g} \cdot \mathrm{ml}^{-1}\right)$ were grafted and after this the sensor was rinsed with TBS.

- Next, the functionalized surface was blocked with bovine serum albumin (BSA), rinsing with TBS.

- After the rate of the syringe pump was reduced to $2 \mu \mathrm{l} / \mathrm{min}$.

- And finally $150 \mu \mathrm{l}$ of the target phages (M13) were injected. 
Furthermore, for some concentrations, the secondary antibody (AM13 $50 \mu \mathrm{g} \cdot \mathrm{ml}^{-1}$ ) was introduced, with a rate of $10 \mu \mathrm{l} / \mathrm{min}$.

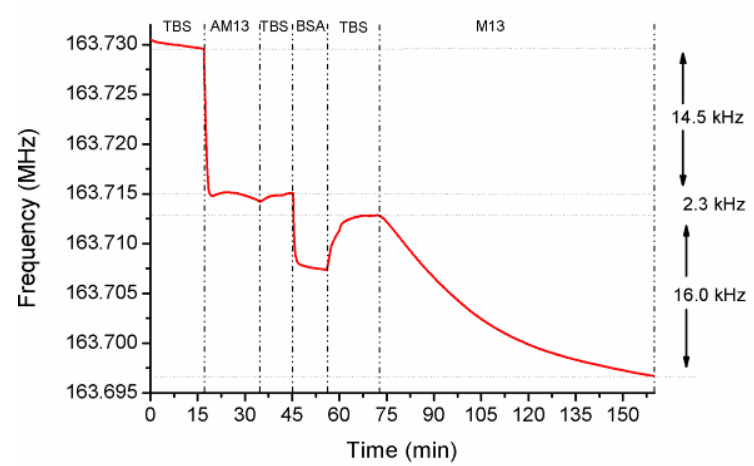

Fig. 3. Frequency response of the following steps: i) $50 \mu \mathrm{g} \cdot \mathrm{ml}^{-1}$ of $\mathrm{AM13}$, ii) $\mathrm{BSA}$ and iii) $2 \times 10^{10}$ phages $\cdot m \Gamma^{1}$, rinsing with TBS between steps.

After of the measurement, the bond between antibodies and phages was weakened with $0.1 \mathrm{M}$ triethylamine, and this allowed extract the phages and quantify them by means of DLA technique that consist of incubating with freshly E. coli (XI1Blue) grown at $37^{\circ} \mathrm{C}$. It is plated in a second layer of soft agar onto a petri dish on top of $30 \mathrm{ml}$ of hard agar. After overnight incubation at the plaques are counted and phage number in plaques-forming unit (pfu) per $\mathrm{ml}^{-1}$ is determined.

\section{Results}

Concentrations between $2 \times 10^{10} \mathrm{pfu} \cdot \mathrm{ml}^{-1}$ and $5 \times 10^{8} \mathrm{pfu} \cdot \mathrm{ml}^{-1}$ of $\mathrm{M} 13$ were measured and discriminated directly through the antibody AM13 (Fig. 4). Each one was repeated three times with similar frequency drop.

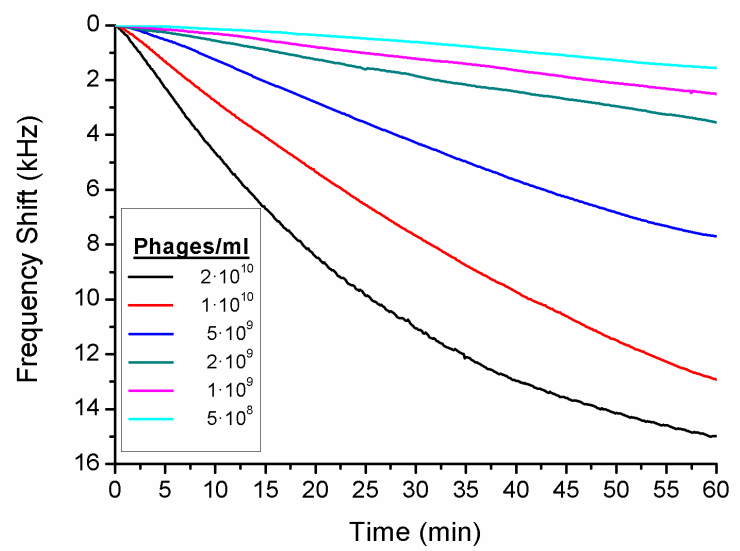

Fig. 4. Frequency shift for the measurements in real time of the different concentrations of the bacteriophage M13.

In order to validate the results concentrations between $2 \times 10^{10} \mathrm{pfu} \cdot \mathrm{ml}^{-1}$ and $2 \times 10^{9} \mathrm{pfu} \cdot \mathrm{ml}^{-1}$ of the phages were detected with GAM antibody as control, obtaining a lower frequency shift due to its low-affinity for this type of phage (Fig. 5).

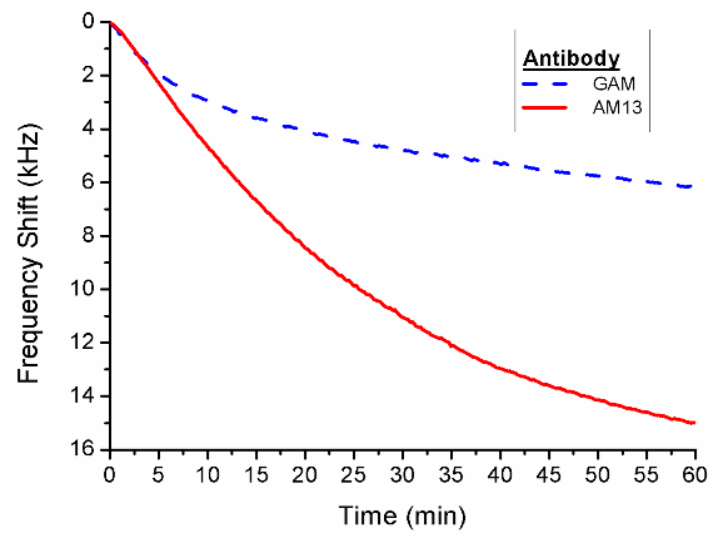

Fig. 5. Comparative measurements between the antibodies AM13 (high affinity) and the GAM ones (low affinity) for the concentration of $2 \times 10^{10} \mathrm{pfu} \cdot \mathrm{ml}^{-1}$.

To obtain a relationship between concentrations of phages detected using the AM13 and the frequency shift, the average of the frequency drop for three measurements at the same concentration (Fig. 6) has been calculated. The values for the different concentrations were adjusted by Langmuir equation. The frequency shift due to phages that have been detected by the antibodies AM13 and GAM has been also compared, being lower for the last one.

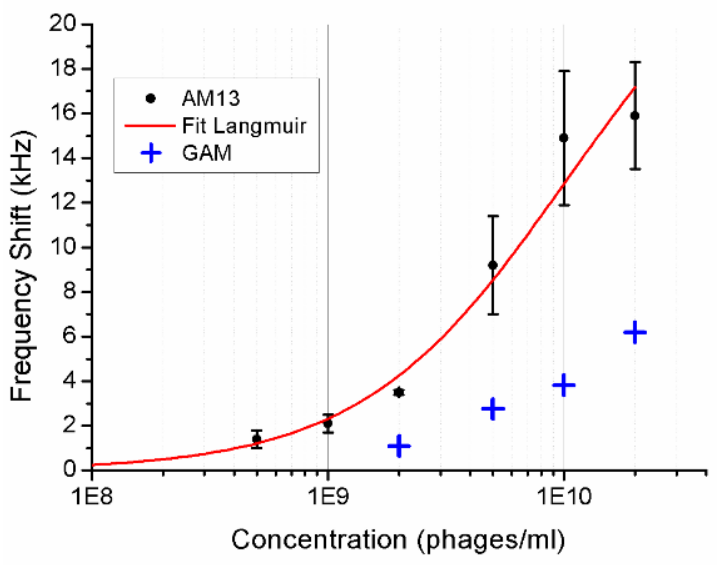

Fig. 6. Frequency shift due to different concentrations of the phage M13. (•) average of three different detections with the antibody AM13, which were fitted by Langmuir model and (+) detected by GAM.

Due to the 2800 proteins of the coating, after the phage immobilization, a lot of antibodies can bind with the unlinked proteins, and it happens in a short time due to the high concentration of these ones $\left(50 \mu \mathrm{g} \cdot \mathrm{ml}^{-1}\right)$, this 
fact was used to measure lower concentrations of phages obtaining a frequency drop about 1 $\mathrm{kHz}$ for $5 \cdot 10^{7}$ pfu. $\mathrm{ml}^{-1}$.

The sensitivity of the Love-wave can be calculated by means of:

$S_{m}^{f}=\frac{\Delta f}{f_{0}} \frac{1}{\rho \cdot d}$

with $\Delta f$ the frequency shift, $f_{0}$ the synchronous frequency, $\rho$ the density of the layer deposited on sensor surface, and $d$ the thickness of the layer. In this way, the difference between two thicknesses (Table 1) of the guiding layer has been used in order to be calculated approximately the mass sensitivity, obtaining $\mathrm{S}_{\mathrm{m}}^{\mathrm{f}}=32 \mathrm{~m}^{2} \cdot \mathrm{Kg}^{-1}$.

Tab. 1: Properties of two different Love-wave devices with different guiding layer thickness (device 1 is the configuration chosen for this experiment).

\begin{tabular}{|l|c|c|}
\hline & $\begin{array}{c}\text { Thickness } \\
\mathrm{SiO}_{2}(\mu \mathrm{m})\end{array}$ & $\begin{array}{c}\text { Synchronous } \\
\text { frequency }(\mathrm{MHz})\end{array}$ \\
\hline Device 1 & 3.5 & 163.200 \\
\hline Device 2 & 3.8 & 159.725 \\
\hline
\end{tabular}

The phages linked to the sensor surface after two different measurements were counted by DLA. The results were similar to those calculated for sensitivity and frequency drop (Table 2)

Tab. 2: Comparison of the phages linked to the sensor surface. On one hand they are counted by $D L A$ as plaques forming unit and on the other hand they are calculated for sensitivity and frequency drop.

\begin{tabular}{|c|c|c|}
\hline & Sample 1 & Sample 2 \\
\hline $\begin{array}{c}\text { Sample } \\
\text { concentration } \\
\left(\text { pfu } \cdot \mathrm{ml}^{-1}\right)\end{array}$ & $10^{10}$ & $10^{9}$ \\
\hline $\begin{array}{c}\text { Frequency shift } \\
(\mathrm{kHz})\end{array}$ & 13 & 2 \\
\hline $\begin{array}{c}\text { Phages counted by } \\
\text { DLA (pfu) }\end{array}$ & $3.5 \cdot 10^{7}$ & $7.5 \cdot 10^{6}$ \\
\hline $\begin{array}{c}\text { Number of phages } \\
\text { calculated by }{ }_{\mathrm{m}}\end{array}$ & $5.6 \cdot 10^{7}$ & $8.6 \cdot 10^{6}$ \\
\hline
\end{tabular}

\section{Conclusions}

A reusable Love-wave immunosensor developed with microfluidics structures has shown high sensitivity and good reproducibility for the low concentrations $\left(2 \times 10^{10} \mathrm{pfu} \cdot \mathrm{ml}^{-1}\right.$ and $5 \times 10^{8} \mathrm{pfu} \cdot \mathrm{ml}^{-1}$ ) detected of the phage M13. In addition it has been possible discriminate them in dynamic mode with a fast response time.

An efficient identification by the monoclonal antibody AM13 has been achieved compared to the identification by the antibody GAM.

Similar results were obtained for the number of phages immobilized on the surface, counted in $\mathrm{pfu} \cdot \mathrm{ml}^{-1}$ by DLA and calculated by sensitivity and frequency drop.

\section{Acknowledgment}

This work has been supported by the Spanish Science and Innovation Ministry under the project TEC2010-21357-C05-04.

\section{References}

[1] M.J. Oliver, et al., Colloids and Surfaces B: Biointerfaces, Vol. 88, Issue 1, 1 November 2011, Pages 191-195.

[2] D. Matatagui, et. al, Talanta, Volume 85, Issue 3, 15 September 2011, Pages 1442-1447.

[3] Lee Makowski, Journal of Molecular Biology, Vol. 228, Issue 3, 5 December 1992, Pages 885-892.

[4] O. Tamarin, et al., Biosensors and Bioelectronics, Vol. 18, Issues 5-6, May 2003, Pages 755-763.

[5] Z. Wang and J. D. N. Cheeke, (1994) Applied Physics Letters, 64 (22), pp. 2940-2942. 